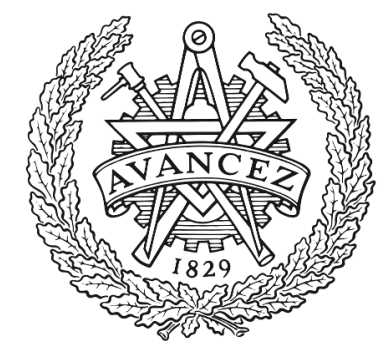

CHALMERS

UNIVERSITY OF TECHNOLOGY

\title{
Feedback Control of Synchronizers for Reducing Impacts during Sleeve to Gear Engagement
}

Downloaded from: https://research.chalmers.se, 2023-04-26 14:16 UTC

Citation for the original published paper (version of record):

Piracha, M., Grauers, A., Hellsing, J. (2020). Feedback Control of Synchronizers for Reducing Impacts during Sleeve to Gear Engagement. SAE Technical Papers, 2020-April(April).

http://dx.doi.org/10.4271/2020-01-0960

N.B. When citing this work, cite the original published paper. 


\title{
Feedback Control of Synchronizers for Reducing Impacts during Sleeve to Gear Engagement
}

\author{
Muddassar Zahid Piracha and Anders Grauers Chalmers University of Technology
}

Johan Hellsing CEVT China Euro Vehicle Technology

Citation: Piracha, M.Z., Grauers, A., and Hellsing, J., "Feedback Control of Synchronizers for Reducing Impacts during Sleeve to Gear Engagement," SAE Int. J. Advances \& Curr. Prac. in Mobility 2(4):2067-2080, 2020, doi:10.4271/2020-01-0960.

This article is from WCX 2020 SAE World Congress Experience.

\section{Abstract}

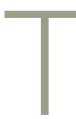

his paper presents a feedback control strategy aimed to reduce noise and wear during gearshifts in conventional and hybrid Dual Clutch Transmissions (DCT and DCTH) and Automated Manual Transmissions (AMT). The control strategy is based on a new dog teeth position sensor developed by China Euro Vehicle Technology AB and existing speed sensors in the transmission. During gear shifting, noise is generated by impacts between the sleeve teeth and the idler gear dog teeth after speed synchronization. Besides noise, these impacts are also responsible for delaying the completion of shift and contribute to wear in the dog teeth, hence reducing the lifespan of the transmission. The presented control strategy controls speed synchronization such that the impact between sleeve and idler gear dog teeth, before the start of torque ramp up, is avoided. Since drag torque is an important factor in speed synchronization, this paper also contains an algorithm to identify friction torque coefficient in the transmission. The identification method ensures that the controller adapts to varying conditions without the need for offline calibration. The control strategy is developed for standard automatic gear shifting operations but minor adaptations in the algorithm also make it capable of handling gear shifts requested by the driver. The output signal of the control strategy is acceleration request on idler gear during speed synchronization. To make controller easier to implement and minimize shift time, the acceleration request only has two values, either maximum value or zero. The control strategy is designed in such a way that it can easily be integrated in the existing transmission control software. By applying the control strategy on a detailed simulation model, it is shown that the impacts during gear engagement are significantly reduced.

\section{Introduction}

(1) hroughout the last decades, reducing emissions has been the focus of the automotive industry. Also, the introduction of stricter legislation during the recent years has made the hybrid powertrains more popular. The introduction of electric motors (EM) for traction in the vehicles have significantly reduced the usage of combustion engines (ICE) so vehicles spend more time driving in zero emission mode. ICE is one of the main sources of noise in the vehicle. When the usage of ICE is decreased other noise sources in the vehicle which were not deemed important in the past become more emphasized.

This paper deals with noise generation during gear shifting. For gear shifting in hybrid vehicles DCT and AMT are used. DCT and AMT use conventional synchronization systems, operated by automated shifting actuators as shown in [1] and [2].

The high-level gear shifting process can be divided into following phases [1].

1. Torque ramp down

2. Sleeve to Neutral
3. Speed Synchronization

4. Sleeve to Gear Engagement

5. Torque Ramp up

At the beginning of gear shifting during torque ramp down phase, driving torque from ICE or traction EM is removed from off going idler gear. Once the torque is zero, sleeve to neutral phase begins, where sleeve is disengaged from offgoing idler and moved to neutral position. In speed synchronization phase the rotational velocity of oncoming idler gear is matched with that of sleeve. Once the speed difference between oncoming idler and sleeve is zero, sleeve to gear engagement phase starts where sleeve is pushed to engage with oncoming idler. After sleeve has moved a certain distance on oncoming idler driving torque from ICE or traction EM is resumed and shift is finished.

The quality of gear shift as perceived by driver is based on the time taken from torque ramp down to torque ramp up [3]. During sleeve to engagement phase, there are impacts between sleeve teeth and idler gear dog teeth [4]. These impacts are responsible for noise during gear shifts in vehicles.

(c) 2020 The Authors. Published by SAE International. This Open Access article is published under the terms of the Creative Commons Attribution License (http://creativecommons.org/licenses/by/4.0/), which permits distribution, and reproduction in any medium, provided that the original author(s) and the source are credited. 
Additionally, these impacts are also responsible for shortening the life span of transmission [4].

A lot of research has been done on dynamic modeling of these impacts as shown in [] ] and []. [7] shows research on control system development to avoid these impacts altogether. In order to avoid the dog teeth impacts, "Dog Teeth Position Sensor" mentioned in [7] is needed. If the performance criterion is only to minimize the gear shift time, the existing speed sensors are enough.

The control system developed in [7] is a model based open loop control system. Since the control action is taken at the start of speed synchronization, the control method in [?] cannot avoid impacts if the system deviates from ideal trajectories during speed synchronization. In this paper, a feedback control strategy that aims to avoid the impacts by continuously monitoring the sensor signals is proposed. Furthermore the model based open loop controller can only minimize the impacts for gear shifts which are part of the standard computer-controlled gear shifting algorithm without modifications. For gear shifts which are requested by driver i.e. Tiptronic shifts, minimization of dog teeth impacts can be achieved by feedback control algorithm much more easily.

During gear shift the feedback controller implementation is kept simple to avoid calculation delays. The complex calculations required for controller are not done during the shift and can be done either offline or when transmission controller is free. The importance of friction torque coefficient in the development of feedback control system is also demonstrated.

An identification method for friction torque coefficient is presented. The method identifies the friction torque coefficient from data collected from gear shifts in the past, under similar conditions. When the same shift is scheduled again under similar conditions, the most accurate value of friction torque coefficient will be available to the feedback controller, hence guarantying accurate control.

The first section of this paper "Simulation Model" describes the speed synchronization and gear engagement phase. In gear engagement phase description, the conditions to avoid contacts between sleeve and idler gear dog teeth are formulated based on [7]. The second section "Feedback control algorithm" describes in detail the design and implementation of the control algorithm. Second section also contains the subsection "Simulation Result" which shows the results of feedback control. Third section is "Adaptation for Tiptronic transmissions" in which the feedback control algorithm is modified so it can handle the driver requested gear shifts. Fourth section is "Identification of friction torque coefficient" in which friction torque coefficient used in "Simulation Model" is identified using Binary Search Algorithm.

\section{Simulation Model}

The principle layout of DCTH is shown in Figure 1. The integrated electric motor (EM) is shown in dark blue color in Figure 1. EM can give traction torque to the vehicle as well as assist during gear shifting. It also increases the inertia of the system which makes speed synchronization more difficult than in a conventional DCT. The gear shift from initial gear ratio to target gear ratio contains two distinct phases.

\section{FIGURE 1 Driveline Model}

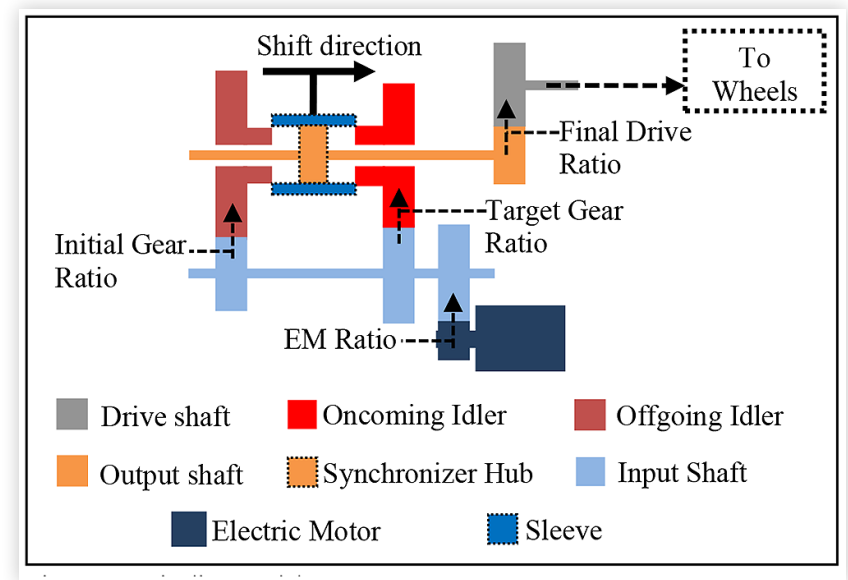

Reprinted with permission from Ref. [7], fig. 1. (c) SAE International.

1. Speed synchronization

2. Gear engagement

\section{Speed Synchronization}

During speed synchronization, the speed of oncoming idler gear $\omega_{g}$ is matched with sleeve speed $\omega_{s}$. As it can be seen from Figure 1, the sleeve is connected to the wheels. In this paper, it is assumed that the shift velocity of vehicle $v_{v e h}$ will remain constant during the shift since the vehicle does not have much time to decelerate if the shift is fast. The angular velocity of driveshaft will be

$$
\omega_{\text {drive shaft }}=v_{\text {veh }} / R_{w}
$$

where $R_{w}$ is the wheel radius.

From $\omega_{\text {drive shaft }}$ in equation $1, \omega_{s}$ can be calculated by

$$
\omega_{s}=\omega_{\text {drive shaft }} \times \text { Final Drive Ratio }
$$

In this paper, the driveshaft is assumed to be infinitely stiff, so there is no torsional degree of freedom between sleeve and wheels.

At the start of speed synchronization, at time $t_{0}$, the velocity of idler gear $\omega_{g 0}$ is calculated by

$$
\omega_{g 0}=\omega_{s} \times(\text { Initial Gear Ratio / Target Gear Ratio })
$$

Relative velocity between sleeve and idler gear is called $\omega_{s g}$, and its initial value $\omega_{s g 0}$, at time $t_{0}$ is

$$
\omega_{s g}\left(t_{0}\right)=\omega_{s g 0}=\omega_{s}-\omega_{g 0}
$$

A synchronization torque $T_{s}$ is then applied on idler gear such that its velocity at synchronization time $t_{\text {synch }}$ is equal to $\omega_{s}$ from equation 2, as shown in Figure 2. So, relative velocity between sleeve and idler gear $\omega_{\text {sg }}$ at time $t_{\text {synch }}$ will be zero.

$$
\omega_{s g}\left(t_{\text {synch }}\right)=0
$$

$T_{s}$ can be provided either by synchronizer ring or by electric motor as explained by [7]. Maximum value of synchronization torque $T_{s}$ provided by either source is denoted by $T_{\max }$, so 


\section{FIGURE 2 Speed synchronization trajectory}

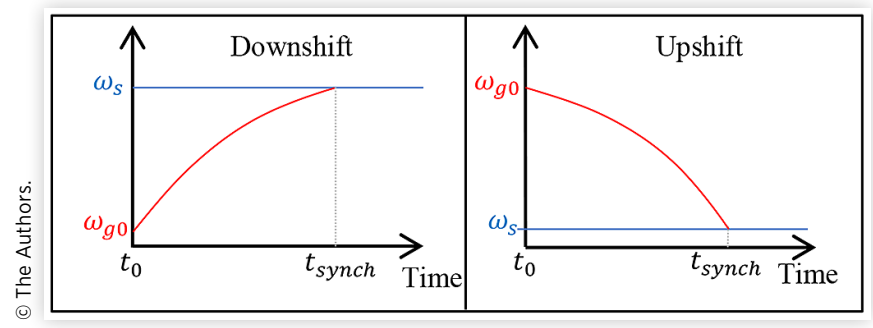

TABLE 1 Speed Synchronization Parameters

\begin{tabular}{|l|l|}
\hline Speed Synchronization Parameters \\
\hline$\omega_{s}$ & $200 \mathrm{rad} / \mathrm{sec}$ \\
$\omega_{g 0}$ & $100 \mathrm{rad} / \mathrm{sec}$ \\
$\omega_{s g 0}$ & $100 \mathrm{rad} / \mathrm{sec}$ \\
\hline$T_{\max }$ & $25 \mathrm{Nm}$ \\
\hline$b$ & $0.1 \mathrm{Nm} / \mathrm{rad} / \mathrm{sec}$ \\
\hline$t_{\text {synch }}$ & $219.7 \mathrm{~ms}$ \\
\hline
\end{tabular}

$$
\max \left(T_{s}\right)=T_{\max }
$$

The rotational dynamics of the idler gear can be written as

$$
J_{g} \dot{\omega}_{g}+b \omega_{g}=T_{s}
$$

where $J_{g}$ is the inertia of idler gear, input shaft and electric motor. Calculation of $J_{g}$ depends on whether the synchronization is done with synchronizer rings or electric motor as explained by [?]. In equation 7, $b$ is a friction torque coefficient and it is derived from physical parameters of synchronizer in $[\underline{8}]$.

The solution of equation 7 at a constant synchronization torque $T_{s}$ for time $t$ where $t \in\left[t_{0}, t_{\text {synch }}\right]$ is

$$
\omega_{g}(t)=\left(T_{s} / b\right)+\left(\omega_{g 0}-T_{s} / b\right) \times e^{-b \times t / I_{g}}
$$

Time $t_{\text {synch }}$ in equation 5 can then be calculated from equation 8 for maximum synchronization torque $T_{\max }$ as

$$
t_{s y n c h}=\ln \left[\left(\omega_{s}-T_{s} / b\right) \div\left(\omega_{g o}-T_{s} / b\right)\right] \times\left(-J_{g} / b\right)
$$

The speed synchronization parameters used in this paper are shown in Table 1.

\section{Teeth Alignment during Speed Synchronization}

A side view and an exploded view of a mechanical synchronizer is shown in Figure 3 . The components in speed synchronization are sleeve, blocker ring and oncoming idler gear as shown in the assembly view in Figure 3.

In Figure 4 the relative positions of the dog teeth of sleeve, blocker ring and idler gear are shown in blocking position as explained by [9], when synchronizer is in speed synchronization phase. The teeth geometry is also shown in Figure 4. The dog teeth width for all teeth is $w_{d o g}$.

\section{FIGURE 3 Mechanical Synchronizer}

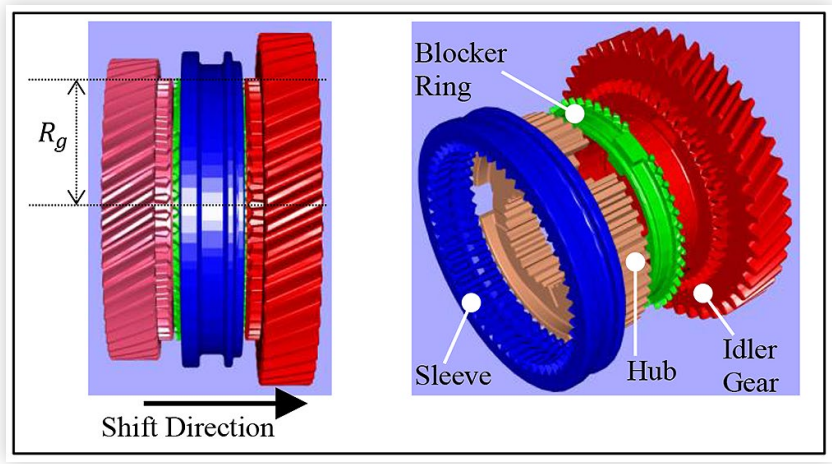

Right half of Figure 3, reprinted with permission from Ref. 7.

\section{FIGURE 4 Synchronizer Teeth during}

Speed synchronization

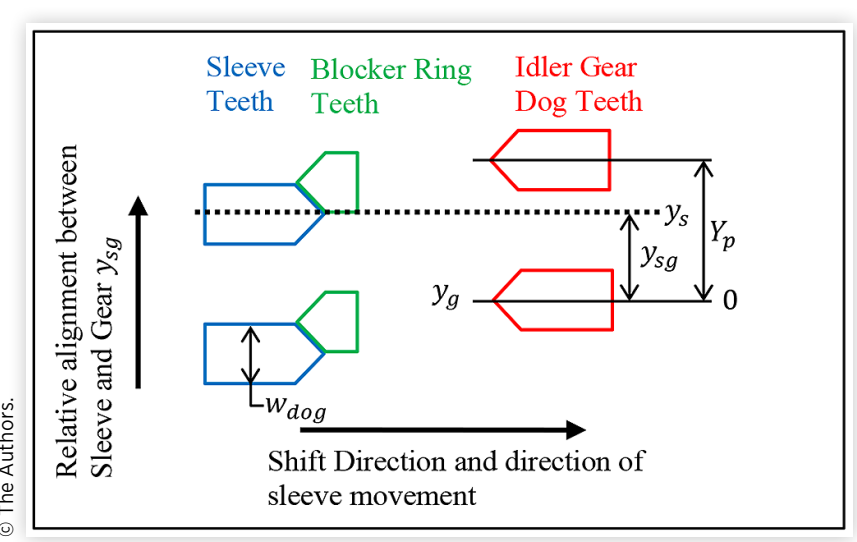

Angular displacement of sleeve $\theta_{s}$ at any time instance $t$ where $t \in\left[t_{0}, t_{\text {synch }}\right]$ can be calculated by integrating constant sleeve velocity $\omega_{s}$ in equation 2 . So, since sleeve velocity is constant during shift

$$
\theta_{s}(t)=\theta_{s 0}+\omega_{s} \times t
$$

where $\theta_{s 0}$ is angular displacement of sleeve at time $t_{0}$.

Teeth alignment of sleeve $y_{s}$ as shown in Figure 4 can then be calculated from equation 10 as

$$
y_{s}(t)=R_{g} \times \theta_{s}(t)
$$

where $R_{g}$ is gear radius as shown in Figure 3

Similarly, angular displacement of idler gear $\theta_{g}$ at any time instance $t \in\left[t_{0}, t_{\text {synch }}\right]$ can be calculated by integrating the idler gear velocity $\omega_{g}$ in equation 8 . So

$$
\theta_{g}(t)=\theta_{g 0}+\frac{T_{s}}{b} \times t+\left(\frac{\omega_{g 0} \times J_{g}}{b}-\frac{T_{s} \times J_{g}}{b^{2}}\right) \times\left(1-e^{-b \times t / J_{g}}\right)
$$

From equation 12 teeth alignment of idler gear $y_{g}(t)$ can be calculated by

$$
y_{g}(t)=R_{g} \times \theta_{g}(t)
$$

Relative teeth alignment between sleeve and idler gear $y_{s g}(t)$ can then be calculated similarly to equation 4 as

$$
y_{s g}(t)=y_{s}(t)-y_{g}(t)
$$




\section{Phase Plane Trajectory in Relative Speed and Alignment}

Speed synchronization can be represented by a phase plane trajectory as shown in Figure 5.

On y-axis in Figure 5 is the relative speed between sleeve and idler gear, and it can be determined from speed sensors in the transmission. The relative speed goes from $\omega_{s g 0}$ in equation 4 to 0 as shown in equation 5 after time $t_{\text {synch }}$ calculated by equation 9 . The direction of arrow shows the evolution of relative speed and relative alignment as time progress.

On $\mathrm{x}$-axis in Figure 5, relative alignment between sleeve and gear is shown. At time $t_{0}$ the relative alignment is $y_{s g}$ and it can be calculated by evaluating equations $11, \underline{13}$ and $\underline{14}$ at time equal to $t_{0}$. So

$$
y_{s g 0}=R_{g} \times\left(\theta_{s 0}-\theta_{g 0}\right)
$$

$\theta_{s 0}$ and $\theta_{g 0}$ in equation 15 are unknown hence making $y_{s g 0}$ unknown. But from [7] it can be seen that $y_{s g}$ can be measured at start of every gear shift by "Dog Teeth Position Sensor". Hence making $y_{s g 0}$ an arbitrary but known constant. Also, from [7] the limits on arbitrary $y_{s g 0}$ can be defined by

$$
y_{s g 0} \in\left[0, Y_{p}\right]
$$

where circular pitch $Y_{p}$ is the distance between two consecutive tips of sleeve or idler gear as shown in Figure 4 and can be calculated by

$$
Y_{p}=2 \pi \times R_{g} / n_{d o g}
$$

where $n_{\operatorname{dog}}$ is the number of dog teeth.

Relative alignment $y_{s g}$ in equation 14 at two time instances, $t_{i}$ and at a later time $t_{i}+\Delta t$ is shown in Figure 6 . It can be seen from Figure 6 that at both time instances $t_{i}$ and $t_{i}+\Delta t$ sleeve dog teeth position is same with respect to idler gear dog teeth. So

$$
\begin{gathered}
\text { if } y_{s g}\left(t_{i}+\Delta t\right)-y_{s g}\left(t_{i}\right)=n \times Y_{p} \\
\text { then } y_{s g}\left(t_{i}+\Delta t\right)==y_{s g}\left(t_{i}\right)
\end{gathered}
$$

where $n$ is a positive integer.

At time $t_{\text {synch }}$ relative alignment is $y_{\text {sg }}\left(t_{\text {synch }}\right)$ and its value will be discussed in the section "Sleeve to gear engagement".

FIGURE 5 Phase plane trajectory for speed synchronization

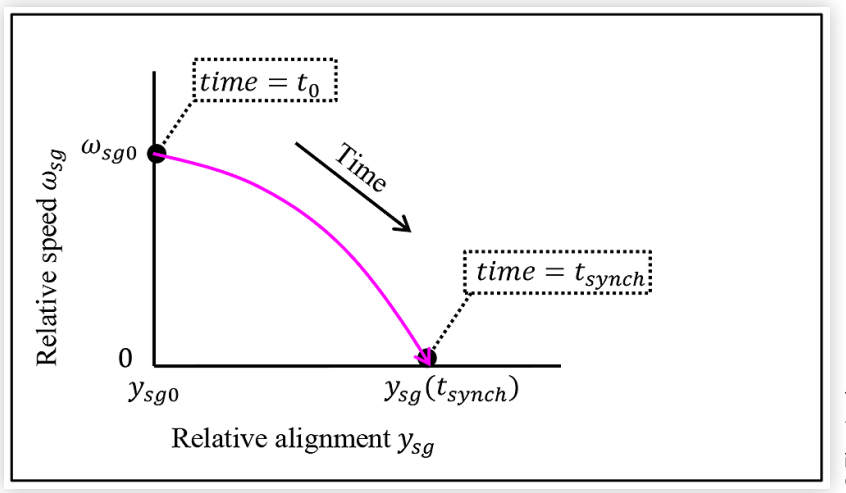

\section{FIGURE 6 Periodicity of $y_{s g}$}

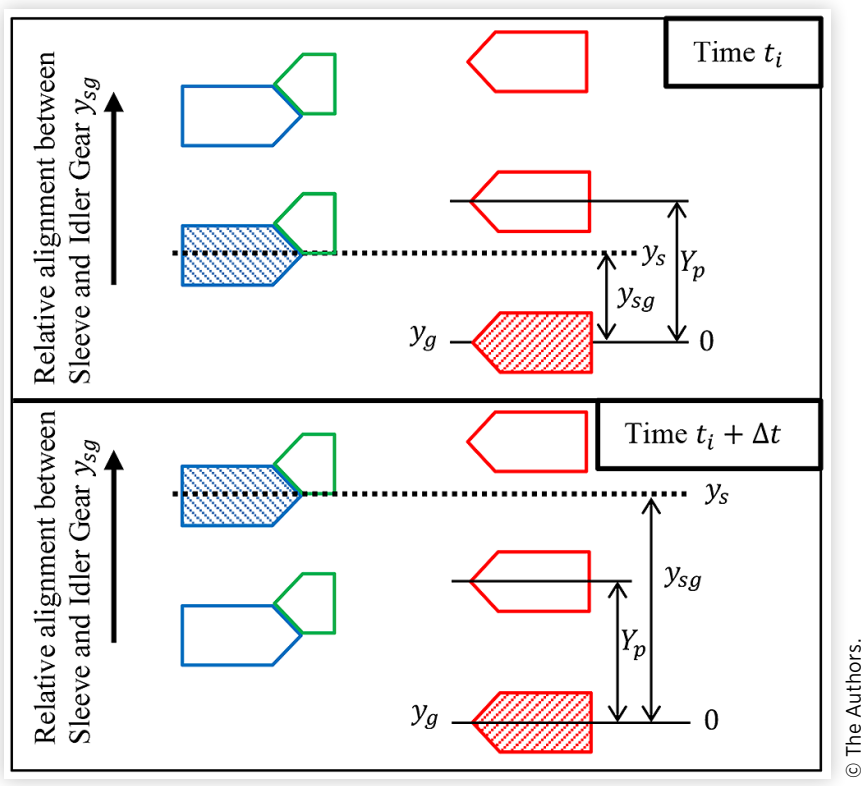

TABLE 2 Teeth Geometry Parameters

\section{Teeth Geometry Parameters}

\begin{tabular}{l|l}
$W_{\text {dog }}$ & $4 \mathrm{~mm}$ \\
$n_{\text {dog }}$ & 45 \\
$Y_{p}$ & $8.5 \mathrm{~mm}$ \\
$R_{g}$ & $60.8768 \mathrm{~mm}$ \\
\hline & Data taken from Ref. [7].
\end{tabular}

The teeth geometry parameters used in this paper are same as used in [7] and are shown in Table 2.

\section{Sleeve to Gear Engagement}

Once speed synchronization is done at time $t_{\text {synch }}$, the sleeve moves towards idler gear as shown in Figure 7. When sleeve has moved a certain displacement $x_{\text {end }}$, the ramp up torque starts at time $t_{\text {end }}$ as shown in Figure 7.

\section{FIGURE 7 Sleeve to Idler gear engagement}

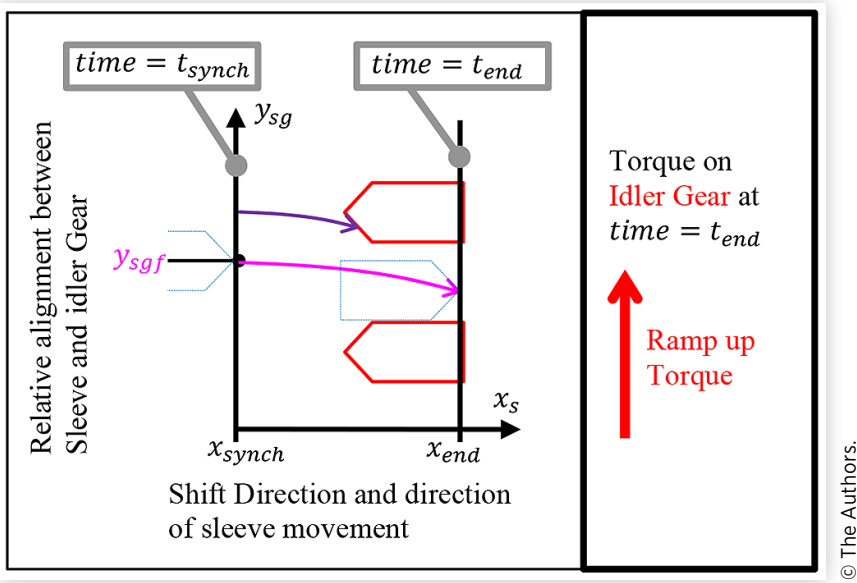


FIGURE 8 Gear engagement time for different $y_{s g}\left(t_{\text {synch }}\right)$

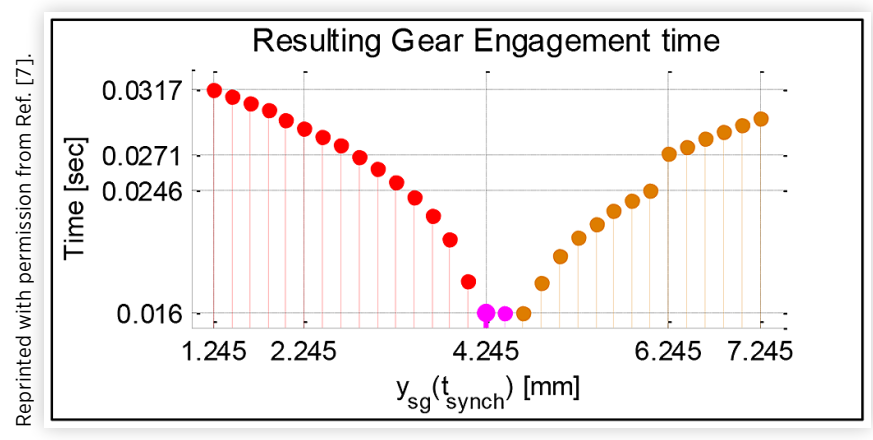

FIGURE 9 Frontal contact force on sleeve for different $\underline{y_{s g}\left(t_{\text {synch }}\right)}$

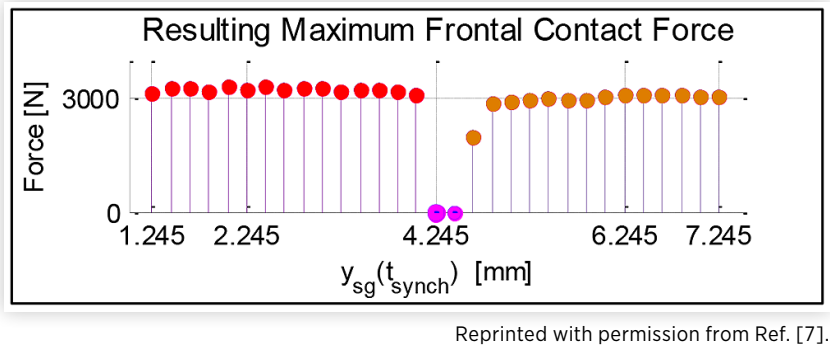

Relative alignment between sleeve and idler gear at synchronization time $y_{s g}\left(t_{\text {synch }}\right)$ determine the trajectory of sleeve tip point during gear engagement as shown by purple and magenta curves in Figure 7. From Figure 7, it can be seen that if $y_{s g}\left(t_{\text {synch }}\right)$ is equal to a particular value $y_{s g f}$ the sleeve teeth do not hit the idler gear dog teeth hence guarantying fastest gear engagement and least noise and wear. If, however gear engagement starts at another value as shown by the purple curve the sleeve teeth make a "frontal contact" which delays gear engagement and makes clonk noise and wear in the transmission. Figure 8 shows the gear engagement time i.e. time it takes the sleeve to travel from $x_{\text {synch }}$ to $x_{\text {end }}$ in Figure $\underline{7}$ for different values of $y_{\text {sg }}\left(t_{\text {synch }}\right)$. Figure 9 shows the frontal contact force experienced by sleeve during gear engagement.

It can be seen from Figures 8 and $\underline{9}$ that if

$$
y_{s g}\left(t_{\text {synch }}\right)=y_{\text {sgf }}=4.245 \mathrm{~mm}
$$

then the gear engagement time and frontal contact force is minimum resulting in fastest engagement with least noise and wear.

[7] shows detailed calculation of $y_{s g f}$ and includes simulation results for verification.

\section{Feedback Control Algorithm}

The control algorithm is applied during speed synchronization phase and the aim of the algorithm is to make $y_{s g}\left(t_{\text {synch }}\right)$ equal to $y_{s g f}$ for any value of $y_{s g 0}$, so that the gear engagement is as fast as possible, with least noise and wear.
The input to transmission during speed synchronization phase is the synchronization torque $T_{s}$. The output signals are relative speed $\omega_{s g}$ coming from speed sensors and relative teeth alignment $y_{s g}$ coming from dog teeth alignment sensors. The top-level diagram of control system is shown in Figure 10.

Although $y_{s g 0}$ will be arbitrary for every shift, there will be a particular value of relative teeth alignment at start of speed synchronization $y_{s g 10}$, such that when maximum synchronization torque $T_{\text {max }}$ is applied on idler gear, the relative alignment at the end of speed synchronization will be $y_{s g f}$ as shown by solid magenta curve in Figure 11. If relative alignment is not $y_{s g 10}$ at start of speed synchronization, at end of speed synchronization relative alignment will not be $y_{s g f}$ as shown by dotted phase plane trajectory in Figure 11.

A zone is defined in the phase plane as shown in Figure 12.

Feedback control is designed such that the phase plane trajectory is kept within the zone. As it can be seen from Figure 12 that the zone converges at $y_{s g f}$ as $\omega_{s g} \rightarrow 0$. The maximum boundary of the zone shown by red curve in Figure 12. The red curve can be defined as a set of values of

\section{FIGURE 10 Feedback controller}

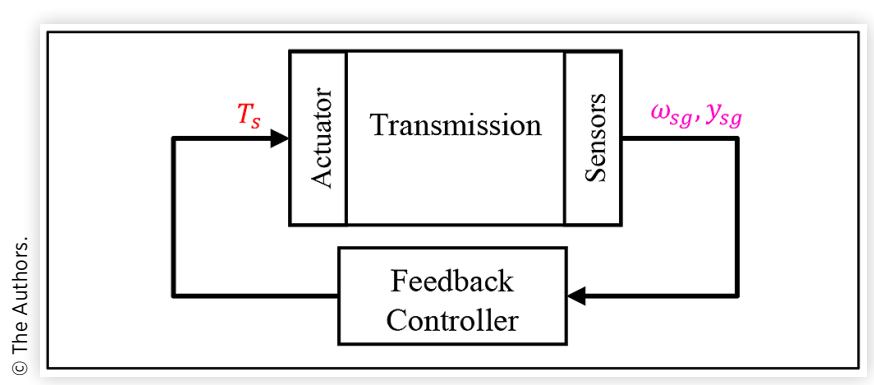

FIGURE 11 Phase plane trajectory for different $y_{s g 0}$

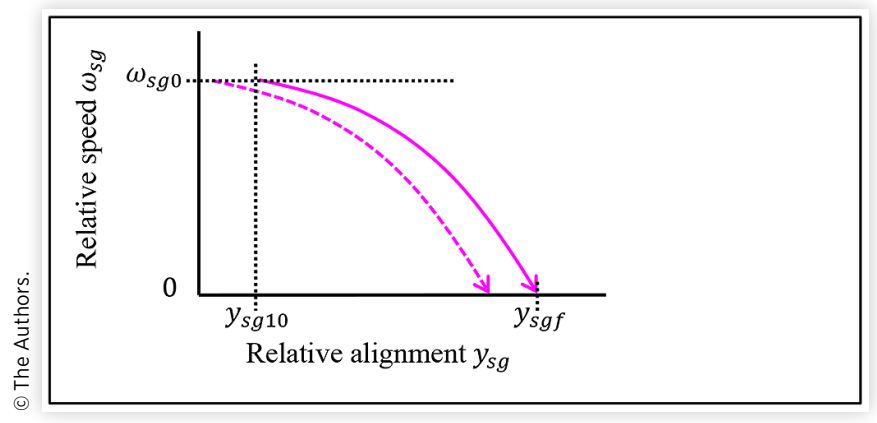

FIGURE 12 Zone for feedback control

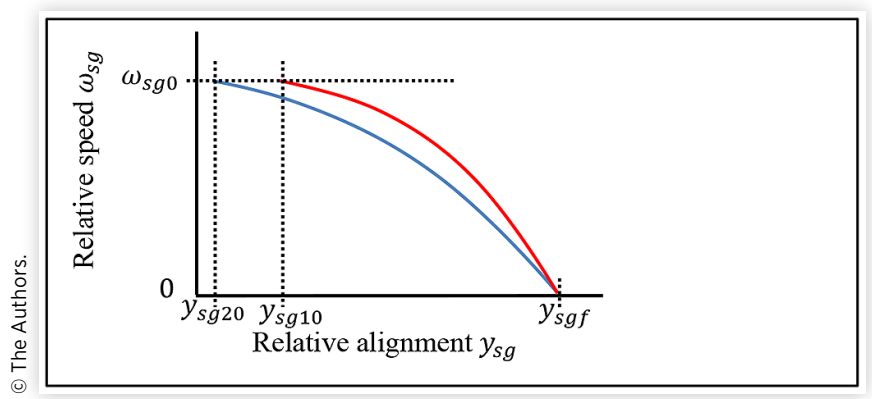


relative alignments $y_{s g 1}$ corresponding to different values of relative velocities $\omega_{s g} \in\left[\omega_{s g}, 0\right]$. So

$$
\text { Maximum Boundary }=y_{s g 1}\left(\omega_{s g}\right)
$$

It can be seen from Figure 12 that the red curve is same as the solid magenta phase plane trajectory in Figure 11. So

$$
y_{s g 1}\left(\omega_{s g 0}\right)=y_{s g 10}
$$

The maximum boundary represents the "Switching Curve" as defined by [10] in the context of time optimal control systems. Once the phase plane trajectory is on the maximum boundary, application of maximum synchronization torque $T_{\max }$, will ensure that relative alignment $y_{s g}$ converges to at $y_{s g}$ as $\omega_{s g} \rightarrow 0$, in minimum time.

The minimum boundary of zone is defined by the blue curve in Figure 12. The blue curve can be defined similarly as a set of values of relative alignments $y_{s g 2}$ corresponding to different values of relative velocities $\omega_{s g} \in\left[\omega_{s g}, 0\right]$. So

$$
\text { Minimum Boundary }=y_{s g 2}\left(\omega_{s g}\right)
$$

From Figure 12 it can be seen that

$$
y_{s g 2}\left(\omega_{s g 0}\right)=y_{s g 20}
$$

Details of calculation of curve $y_{s g 1}$ will be discussed in next section "Generation of Maximum Boundary". The calculation of $y_{s g 2}$ with respect to $y_{s g 1}$ is discussed in subsequent section "Generation of Minimum Boundary".

The feedback controller is designed to keep the phase plane trajectory within the zone shown in Figure 13. It should be noted that during a shift only the simple state-machine shown in Figure 13 is active while the phase plane boundaries are pre-calculated before the shift.

Figure 14 shows the application of feedback control algorithm shown in Figure 13. At start of speed synchronization dog teeth position sensor measures that the relative alignment is $y_{s g 0}$ shown by the magenta dot in Figure 14. Since $y_{s g 0}$ is greater than $y_{\text {sg20 }}$, "Maximum acceleration" state in Figure 13 will be activated. Maximum synchronization torque $T_{\max }$ will be applied to the idler gear and the phase plane trajectory generated by sensor signals shown by dashed magenta curve in Figure 14 will be parallel to the Maximum Boundary shown in red.

\section{FIGURE 13 Feedback Control Algorithm}

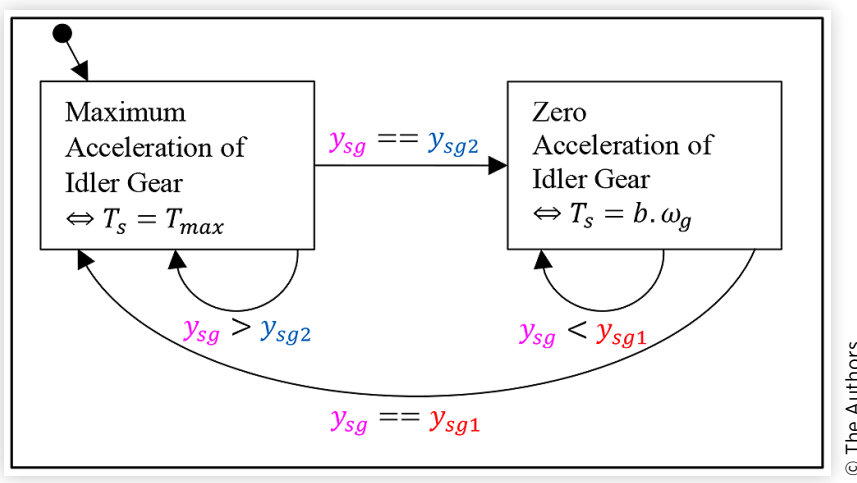

\section{FIGURE 14 Application of feedback controller}

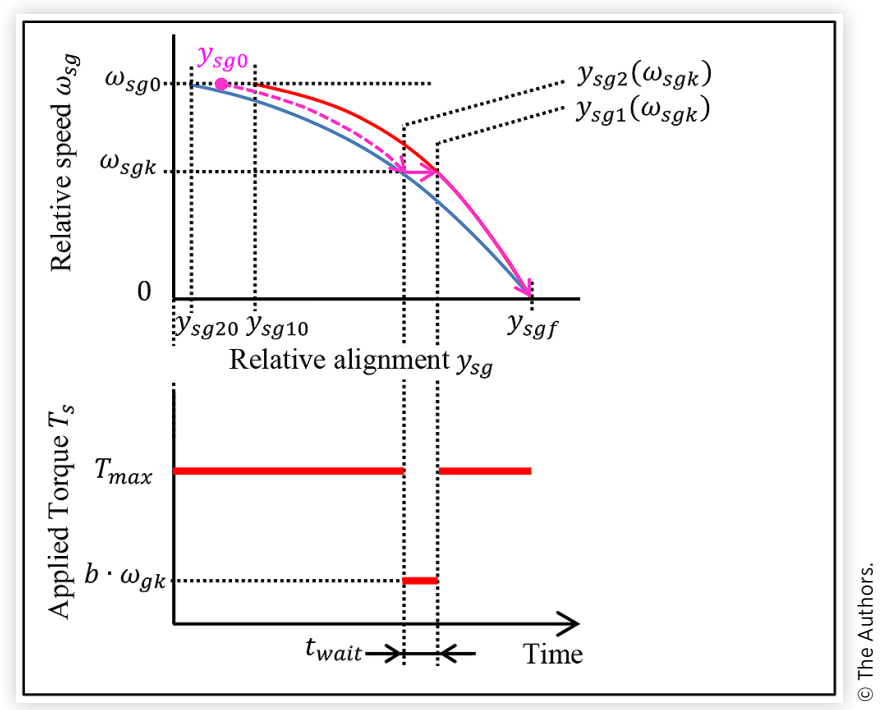

The relative speed $\omega_{s g}$ will keep on decreasing until a particular relative velocity $\omega_{s g k}$. At $\omega_{s g k}$, the phase plane trajectory generated by sensors will hit the Minimum Boundary shown in blue color in Figure 14. Since at $\omega_{s g k}, y_{s g}$ will be equal to $y_{s g 2}$, then according to Figure 13 the state will be switched to "Zero Acceleration" state. Synchronization torque equal to $b \times \omega_{g k}$ will then be applied on idler gear which according to equation 7 will lead to a constant $\omega_{g k}$ and consequently a constant $\omega_{\text {sgk }}$.

After some time $t_{\text {wait }}$, phase plane trajectory generated by sensors will hit the Maximum Boundary i.e. $y_{s g}$ will be equal to $y_{s g 1}$. According to Figure $13, y_{s g}$ being equal to $y_{s g 1}$, will make the state switch back to "Maximum acceleration" state. Application of maximum synchronization torque $T_{\max }$ will then make the phase plane trajectory generated by sensors follow the maximum boundary guaranteeing that when $\omega_{s g} \rightarrow 0, y_{s g} \rightarrow y_{s g f}$

It can be seen from Figure 14 that for feedback controller to work it is essential that

$$
y_{s g 20}<y_{s g 0} \leq y_{s g 10}
$$

Figure 15 shows the situation if the switch to maximum acceleration state when $y_{s g}$ is equal to $y_{s g 1}$ is not made. It would not be possible to reach $y_{s g f}$ as $\omega_{s g} \rightarrow 0$, but, it would still be possible to aim for $y_{s g f}+Y_{p}$ as shown in Figure 15. $y_{s g f}+Y_{p}$

\section{FIGURE 15 Exceeding the Maximum Boundary}

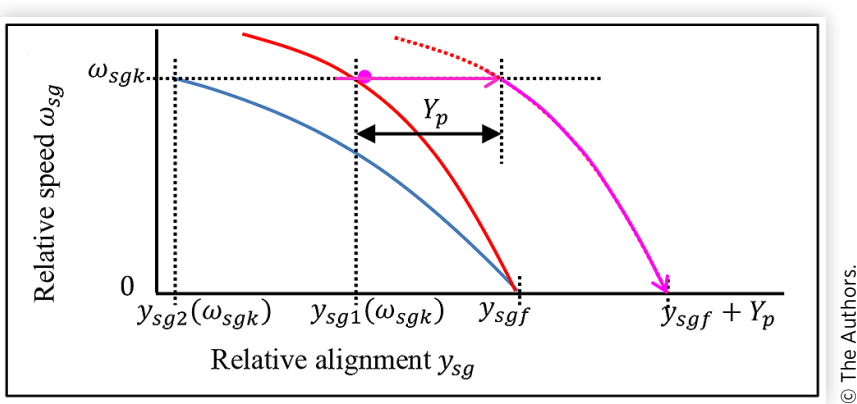


and $y_{s g f}$ represent same relative alignment according to equation 18, so the subsequent gear engagement will still be fastest with least noise and wear. But the synchronization time will be increased, since more time will be spent in zero acceleration state. So, in this paper it is assumed that the state switches shown in Figure 13 are always followed.

\section{Generation of Maximum Boundary}

As mentioned earlier the trajectory $y_{s g 1}$ is generated when maximum synchronization torque $T_{\text {max }}$ is applied on idler gear and the relative alignment at the end of speed synchronization will be $y_{s g f}$ as shown in Figure 11. To calculate Maximum Boundary in equation 20 and $y_{s g 10}$ in equation 21, a backwards in time simulation is run. The algorithm for backwards in time simulation is shown in Figure 16.

As it can be seen in "Initialize" block of Figure 16, Synchronization Torque is set at $T_{\max }$ and $\omega_{g}\left(t_{\text {synch }}\right)$ is initialized according to equation $5, \theta_{s}\left(t_{\text {synch }}\right)$ and $\theta_{g}\left(t_{\text {synch }}\right)$ are initialized such that equation 19 is fulfilled, according to equations $\underline{11}, \underline{13}$ and $\underline{14}$.

The simulation starts at time instance $t_{\text {synch }}$ and runs with a small decremental step of $\delta t$ until time $t_{0}$ as shown in the "Initialize" and "Decrement" blocks of Figure 16.

Idler gear velocity at previous time instance $\omega_{g}\left(t_{i}-\delta t\right)$ is calculated based on idler gear velocity at present time instance $\omega_{g}\left(t_{i}\right)$ according to equation 8 as shown in "Calculate" block of Figure 16. Similarly, angular displacement of idler gear and sleeve at previous time, $\theta_{g}\left(t_{i}-\delta t\right)$ and $\theta_{s}\left(t_{i}-\delta t\right)$ are calculate based on equations 12 and $\underline{10}$ respectively.

After time $t_{\text {synch }}-t_{0}$, "Post Processing" block is activated calculating $\omega_{\text {sg }}(t)$ and $y_{\text {sg_init }}(t), \quad \forall t \in\left[t_{0}, t_{\text {synch }}\right]$. Relative

\section{FIGURE 16 Backwards in time simulation}

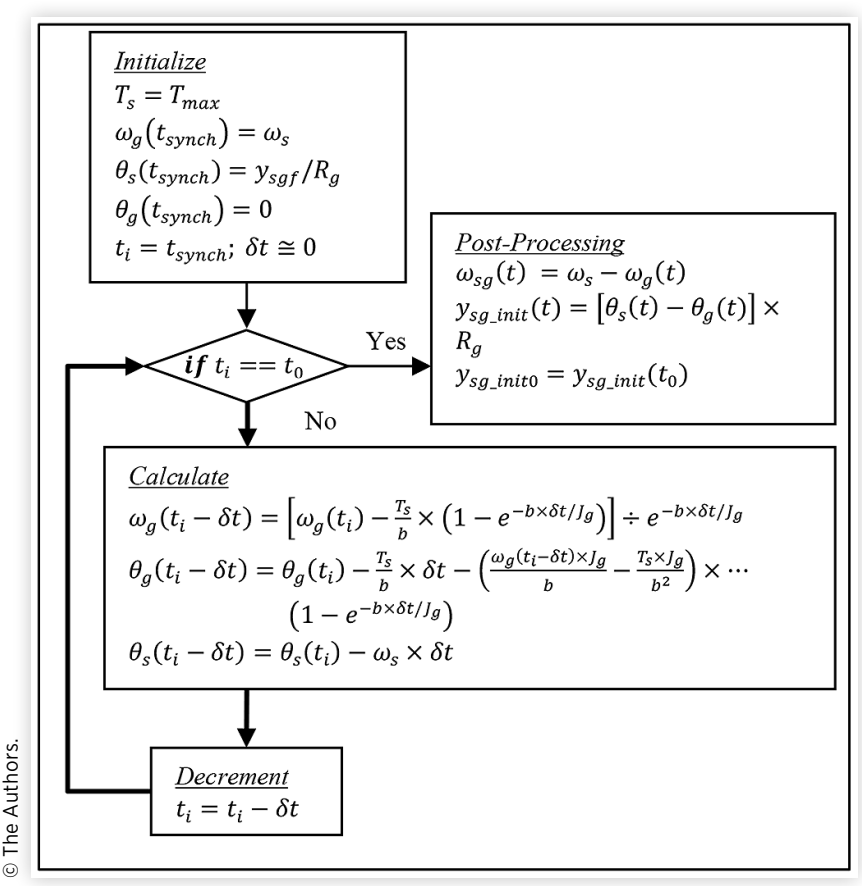

FIGURE 17 Phase plane trajectory from Backwards in time simulation

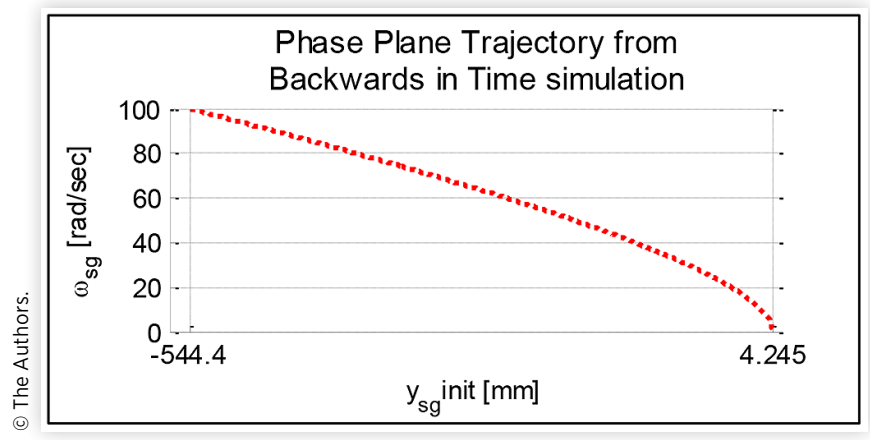

angular alignment at time $t_{0}$ is $y_{\text {sg_init } 0}$ is also calculated as shown in Figure 16.

The resulting phase plane trajectory from Figure 16 based on input parameters in Tables 1 and $\underline{2}$ is shown in Figure 17.

The phase plane trajectory starts at $y_{\text {sg_init } 0}$ equal to $-544.4 \mathrm{~mm}$ and ends at $y_{s g f}$ equal to $4.245 \mathrm{~mm}$ as shown in Figure 17. Now $y_{s g_{i} \text { init } 0} \notin\left[0, Y_{p}\right]$ because $y_{s g f} \in\left[0, Y_{p}\right]$. Also $y_{s g 0} \in\left[0, Y_{p}\right]$ as shown in equation 16. So in order to ensure $y_{s g 0} \leq y_{s g 10}$ condition in equation 24, phase plane trajectory shown in Figure 17 needs to be offset according to condition 18. So

$$
y_{s g 1}(t)=y_{s g_{-} \text {init }}(t)+N \times Y_{p}
$$

Resulting maximum boundary $y_{s g 1} v s . \omega_{s g}$ is shown by solid red curve in Figure 18. The integer $\mathrm{N}$ in equation 25 can be calculated by using ceiling function, which rounds a number to nearest largest integer.

$$
N=\operatorname{ceil}\left[\left(y_{s g 0}-y_{s g_{-} \text {init } 0}\right) \div Y_{p}\right]
$$

$y_{\text {sg10 }}$ can be calculated by evaluating equation 25 at time $t$ equal to $t_{0}$. So

$$
y_{s g 10}=y_{s g_{-} \text {init } 0}+N \times Y_{p}
$$

The ceiling function in equation 26 together with equation 27 ensures $y_{s g 0} \leq y_{s g 10}$ condition in equation 24 is fulfilled.

\section{FIGURE 18 Maximum Boundary and result of backwards in} time simulation

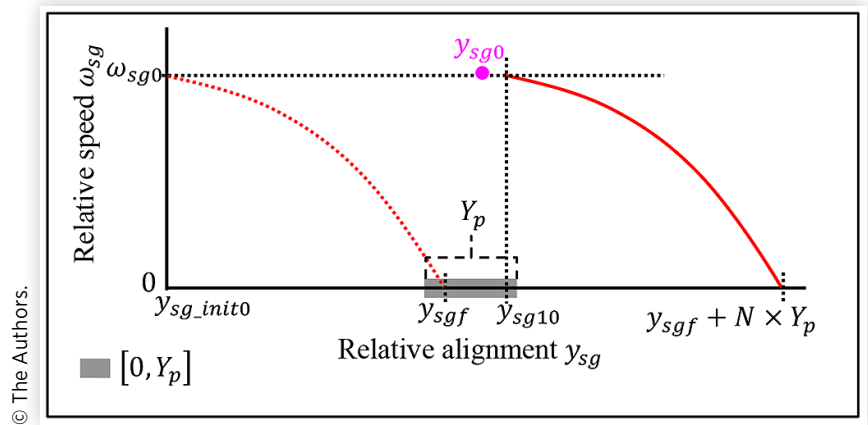




\section{Generation of Minimum Boundary}

Minimum Boundary in equation 22 can be defined as a curve different from Maximum Boundary $y_{s g 1}$ by difference $y_{s g \_d i f f}$. So

$$
y_{s g 2}=y_{s g 1}-y_{s g_{-} d i f f}
$$

The difference $y_{s g_{-} \text {diff }}$ between maximum and minimum boundaries can be defined as a function of relative velocity $\omega_{s g}$ as shown on left side of Figure 19. Since the zone converges to $y_{s g f}$ as $\omega_{s g} \rightarrow 0$, so

$$
y_{s g_{-} \text {diff }}=0 \text { at } \omega_{s g}=0
$$

as shown on left side in Figure 19.

According to equation $16 y_{s g 0} \in\left[0, Y_{p}\right]$, value of $y_{s g 20}$ to ensure the condition $y_{s g 20}$ is less than $y_{s g 0}$ in equation 24 will be if

$$
\begin{gathered}
\text { at } \omega_{s g}=\omega_{s g 0} \\
y_{s g_{-} \text {diff }}=y_{s g 10}-y_{s g 20}=Y_{d}
\end{gathered}
$$

where

$$
Y_{d} \geq Y_{p}
$$

The upper limit of $Y_{d}$ will be discussed in later section "Increase in synchronization time due to feedback control".

To describe $y_{s g_{-} \text {diff }}$ as function of $\omega_{s g}$ a straight line is drawn between two points described by equations 29 and $\underline{30}$ as shown by orange line in left side of Figure 19. The equation of line will then be

$$
y_{s g_{-} \text {diff }}=Y_{d} \times \frac{\omega_{s g}}{\omega_{s g 0}}
$$

The resulting $y_{s g 2}$ after application of equation 28 is shown in Figure 19 on right side.

The zone generated from phase plane trajectory shown in Figure 17 with arbitrary parameters shown in Table 3 is shown in Figure 20.

Based on equation 26, for $y_{s g 0}$ equal to $2 \mathrm{~mm}$ and $y_{\text {sg_init } 0}$ equal to $-544.4 \mathrm{~mm}, N$ will be 65 . From Figure 20 it can be seen that $556.245 \mathrm{~mm}=y_{s g f}+N \times Y_{p}$, so if feedback control can make $y_{s g} \rightarrow 556.245 \mathrm{~mm}$ as $\omega_{s g} \rightarrow 0$, for an arbitrary value of $y_{s g 0}$, in this case $2 \mathrm{~mm}$ the subsequent gear engagement will be the fastest engagement with least noise and wear.

\section{FIGURE 19 Generation of Minimum Boundary}

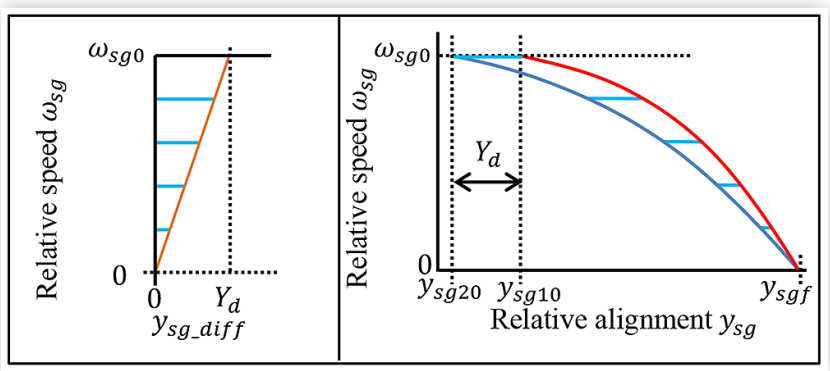

TABLE 3 Arbitrary shift parameters

\section{Arbitrary shift parameters}

\begin{tabular}{l|l}
$Y_{d}$ & $20 \mathrm{~mm}$ \\
$y_{s g 0}$ & $2 \mathrm{~mm}$
\end{tabular}

(c) The Authors.

FIGURE 20 Zone for feedback control based on backwards in time simulation

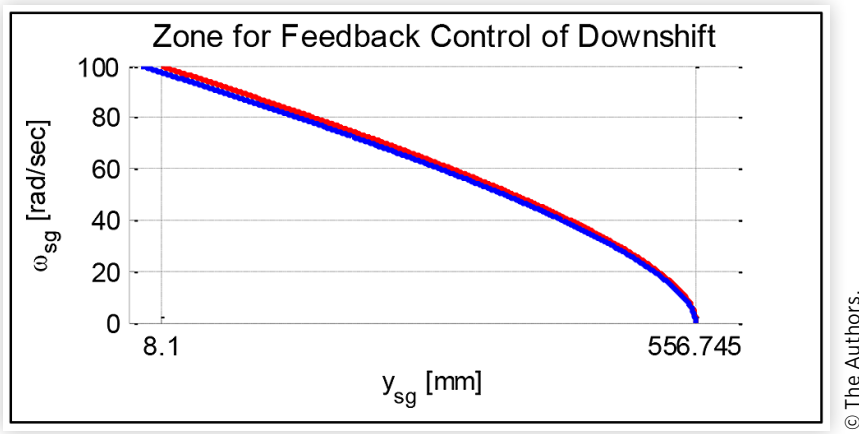

\section{Simulation Result for Feedback Controller}

Phase plane trajectory with application of feedback control with shift parameters shown in Table 3 , is shown in Figure 21a.

In Figure 21, the magenta phase plane trajectory is generated by sensor signals. Red and blue phase plane trajectories are the maximum and minimum boundaries respectively.

\section{FIGURE 21 Simulation result for feedback controller}

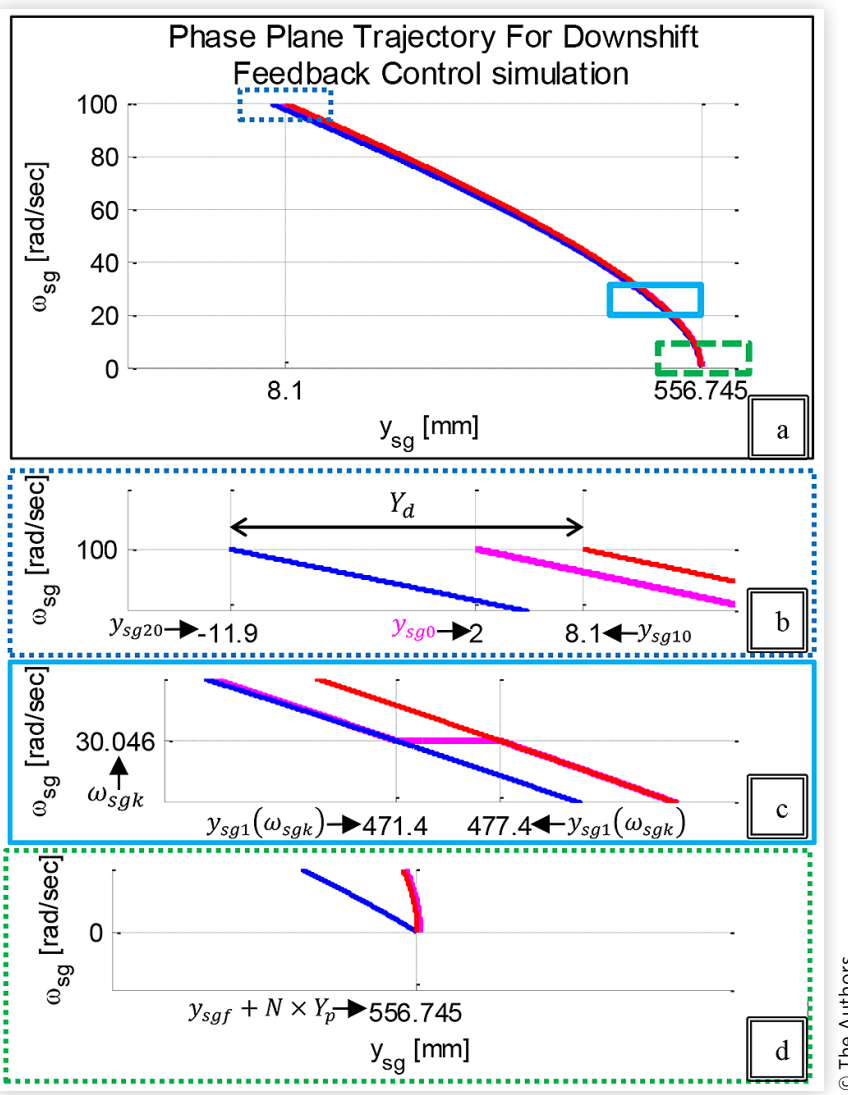


From Figure $21 \mathrm{~b}$ it can be seen that $y_{s g}$ is $2 \mathrm{~mm}$. Since the starting state as defined by Figure 13 is in "Maximum Acceleration" state, the magenta phase plane trajectory travels in parallel with Maximum boundary. At relative speed $30.046 \mathrm{rad} / \mathrm{sec}$, the magenta phase plane trajectory hits the Minimum boundary as shown in Figure 21b. The control algorithm shown in Figure 13, then switches the state to "Zero Acceleration" resulting in constant speed of magenta curve shown in Figure 21b. The state is switched back to "Maximum Acceleration when the magenta curve hits the "Maximum boundary". Figure 21 shows the convergence of magenta phase plane trajectory at the desired $y_{s g f}$ as $\omega_{s g} \rightarrow 0$.

\section{Increase in Synchronization Time due to Feedback Control}

Application of feedback control algorithm shown in Figure $\underline{13}$ changes the speed synchronization trajectory shown in Figure 2 as shown in Figure 22 for downshift.

From Figure 22 it can be seen that application of the feedback control algorithm increases the synchronization time by time $t_{\text {wait }}$. Time $t_{\text {wait }}$ in Figure 22 can be calculated from Figure 14 as

$$
t_{\text {wait }}=\left[y_{s g 1}\left(\omega_{s g k}\right)-y_{s g 2}\left(\omega_{s g k}\right)\right] \div\left[R_{g} \times \omega_{s g k}\right]
$$

Value of $t_{\text {wait }}$ calculated from equation 33 , using numerical values from Figure 22 is $32.8 \mathrm{~ms}$.

The numerator in equation 33 is equal to $y_{\text {sg_diff }}$ according to equation 28 and after substituting the value of $y_{s g \_d i f f}$ from equation 32, the expression of $t_{\text {wait }}$ can be written as

$$
t_{\text {wait }}=Y_{d} \div\left(\omega_{s g 0} \times R_{g}\right)
$$

Equation 34 shows that $t_{\text {wait }}$ is constant and independent of $\omega_{s g k}$. Using minimum value of $Y_{d}$ i.e. $Y_{p}$ according to equation 31, minimum $t_{\text {wait }}$ can be formulated as

$$
\text { minimum }_{\text {wait }}=Y_{p} \div\left(\omega_{s g} \times R_{g}\right)
$$

Value of minimum $t_{\text {wait }} \cong 1.4 \mathrm{~ms}$ based on parameters in Tables 1 and $\underline{2}$, which is very small as compared to $t_{\text {synch }}$ i.e. $219.7 \mathrm{~ms}$.

According to Figure 14 time $t_{\text {wait }}$ is the time where feedback controller changes state according to Figure 13. In this paper, it is assumed that the synchronization torque can change between two values $T_{\max }$ and $b \times \omega_{g k}$ instantaneously

\section{FIGURE 22 Speed synchronization trajectory with} feedback control

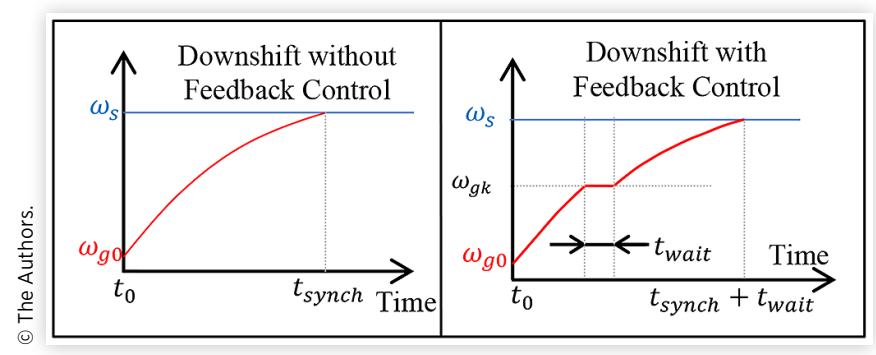

\section{FIGURE 23 Torque output from controller}

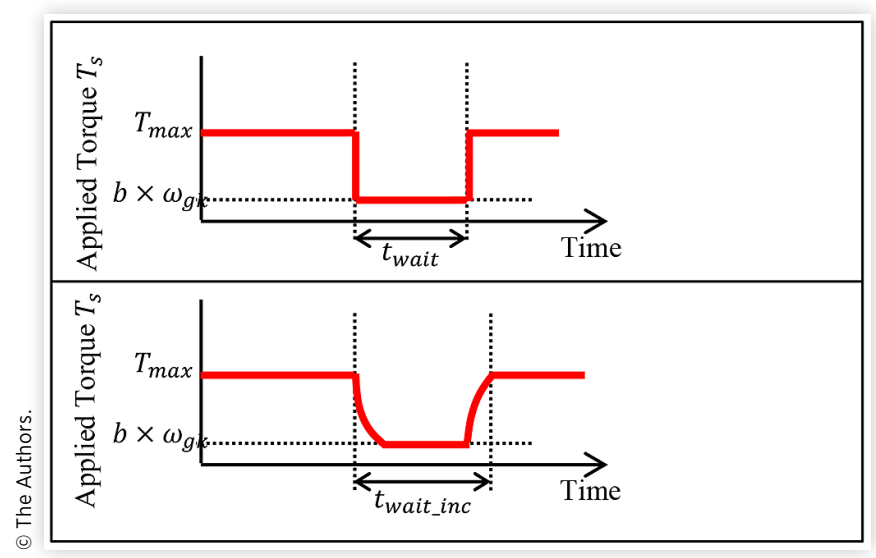

as shown by red lines in upper half of Figure 23, but, due to actuator delays and communication delays in the control system, this would not be the case. More realistic synchronization torque is shown by red lines in lower half of Figure 23 .

If $t_{\text {wait_inc }}$ is the total wait time taking account of before mentioned delays as shown in lower half of Figure 23, then limits on $Y_{d}$ can be defined using equation 34 and equation 31. So

$$
Y_{d} \in\left[Y_{p}, t_{\text {wait_inc }} \times \omega_{s g 0} \times R_{g}\right]
$$

In this paper, actuator delays and communication delays are ignored. Their inclusion in the feedback control can be a topic of future research. It should be noted that, even with delays and actuator dynamics taken into account, the phase plane trajectory generated by intermediate values of torque shown in Figure 23 can still be calculated using equations 8,10 and $\underline{12}$, but will have minor change in its shape as compared to Figure 14, so Maximum and Minimum boundary definitions need to be updated accordingly.

\section{Linear and Exponential Convergence}

Description of $y_{\text {sg_diff }}$ according to equation 32 represents linear convergence of zone towards $y_{s g f}$ as $\omega_{s g} \rightarrow 0$. Instead of a straight line drawn between two points described by equa$\underline{\text { tions } 29}$ and $\underline{30}$ any other curve can also be drawn. For instance an exponential curve $y_{s g_{\text {_diff }}}$, can be drawn as shown by the green curve in Figure 24. Then $y_{\text {sg_diff } 2}$ will represent an

\section{FIGURE 24 Linear and Exponential Convergence}

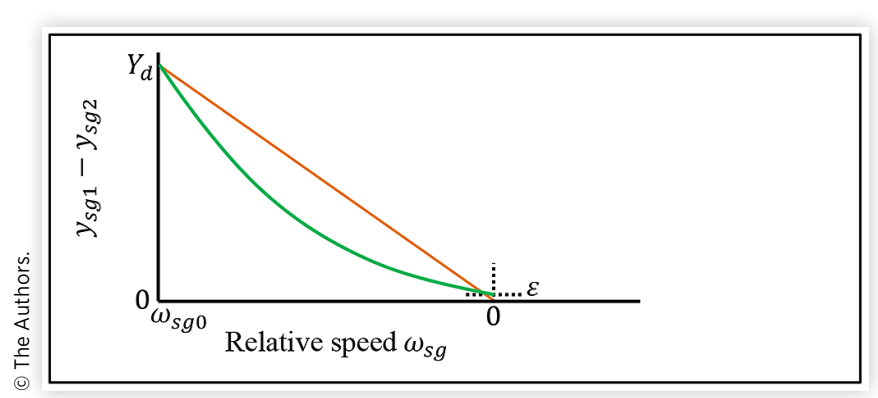




\section{FIGURE $25 t_{\text {wait }}$ for Linear and exponential convergence}

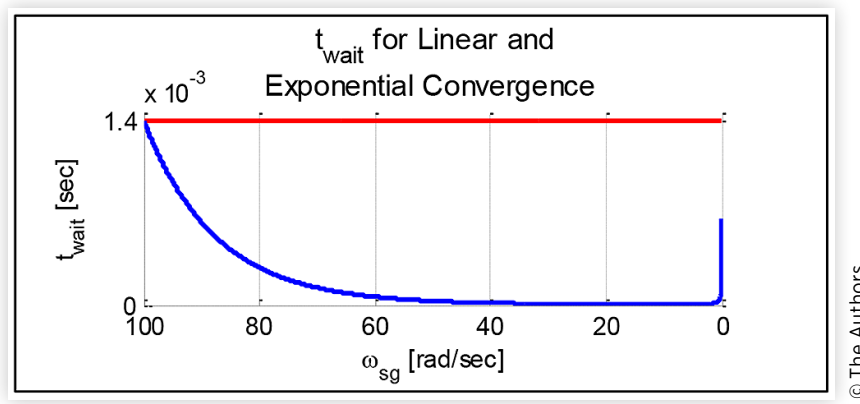

exponential convergence of zone towards $y_{s g f}$ as $\omega_{s g} \rightarrow 0$. In Figure 24, similar to equation $30, y_{s g \_ \text {diff } 2}$ is equal to $Y_{d}$ at $\omega_{s g}$ equal to $\omega_{s g 0}$. But as shown in Figure 24

$$
y_{s g_{-} \text {diff } 2}=\varepsilon \text { at } \omega_{s g}=0
$$

where $\varepsilon$ is a very small positive number.

The equation of the exponential curve $y_{\text {sg_diff } 2}$ can then be written as a function of relative speed $\omega_{s g}$ as

$$
y_{s g_{-} \text {diff } 2}=\varepsilon \times\left(e^{\frac{\ln \left(\frac{Y_{d}}{\varepsilon}\right)}{\omega_{s g 0}}}\right)^{\omega_{s g}}
$$

The justification of $\varepsilon>0$ is shown by equation 38 , where if $\varepsilon=0 y_{\text {sg_diff } 2}$ cannot be calculated.

Use of exponential curve in the feedback controller will be a topic of future research. In the rest of this paper linear curve is used, but the effect of using linear and exponential curves on $t_{\text {wait }}$ can be evaluated using equation 33 , and substituting numerator with $y_{\text {sg_diffi }}$. Value of $t_{\text {wait }} v s . \omega_{\text {sg }}$ for both convergences with $\varepsilon=1 e-3 \mathrm{~mm}$ shown in Figure 25

It can be seen from Figure 25 that $t_{\text {wait }} \rightarrow 0$, if the state switch is done at low values of $\omega_{s g}$. This will in theory decrease $t_{\text {wait }}$ significantly but synchronization torque will not be able to switch in such a small time.

\section{Adaptation for Tiptronic Transmissions}

In Tiptronic transmissions the driver can request upshift or downshift at shift speeds other than $v_{\text {veh }}$ in equation 1 . For these transmissions, the value of relative speed between sleeve and gear before speed synchronization $\omega_{s g 0}$ is not known beforehand. To avoid engine over revving, for downshifts there is a maximum speed above which the shift will not be initiated on driver's request. Similarly, to avoid engine stalling there is a minimum speed below which the up shift will not be initiated on driver's request.

If a downshift is requested by the driver at a vehicle speed greater than $v_{v e h}$, the corresponding shift velocity will also be larger than $\omega_{s g 0}$ as shown in Figure 26.

To adapt the feedback algorithm for Tiptronic transmissions, first maximum synchronization torque $T_{\max }$ will

\section{FIGURE 26 Tiptronic Downshift}

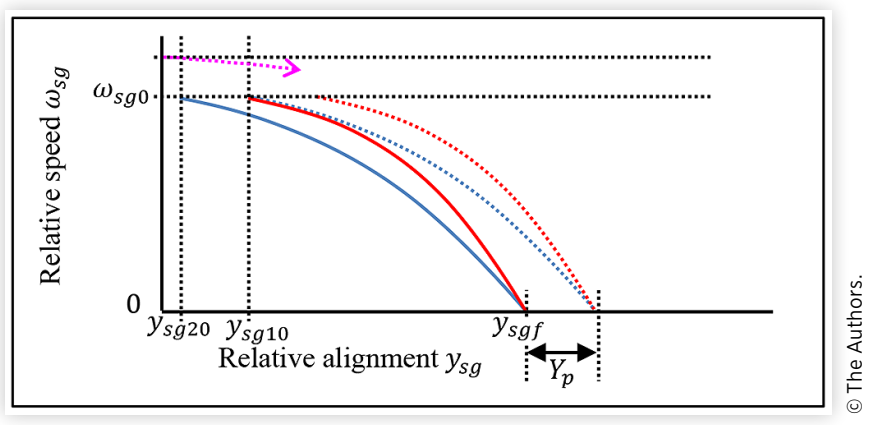

\section{FIGURE 27 Feedback control of Tiptronic Downshift}

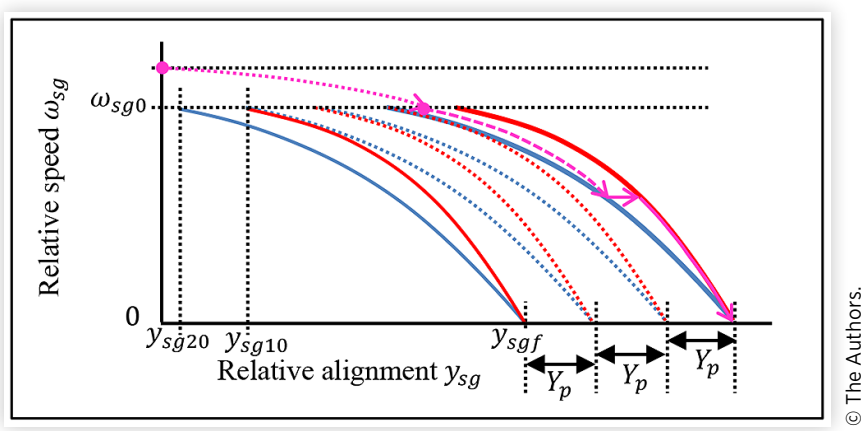

be applied to the idler gear. Consequently $\omega_{s g}$ will start decreasing. $y_{s g}$ from dog teeth position sensor shown in magenta in Figure 26 is continuously read. The zone is offset by $Y_{p}$ by condition

$$
\begin{gathered}
\text { if } y_{s g 0}>y_{s g 10} \\
\text { then } y_{s g 1}=y_{s g 1}+Y_{p} \\
y_{s g 2}=y_{s g 1}-y_{s g_{-} \text {diff }}
\end{gathered}
$$

The offset zone is shown by dotted curves in Figure 26.

As maximum synchronization torque $T_{\max }$ is applied the relative speed $\omega_{s g}$ will keep decreasing until it reaches $\omega_{s g 0}$ as shown in Figure 27.

Meanwhile the zone will be moved left in steps of $Y_{p}$ such that $y_{s g}$ is always between $y_{s g 20}$ and $y_{s g 10}$ as shown in Figure 27. When $\omega_{s g}$ is equal to $\omega_{s g 0}$, the zone offsetting from equation $\underline{39}$ will be such that $y_{s g}$ will satisfy the equation 24 . After this the feedback control algorithm shown in Figure 13 will be applied to make $y_{s g} \rightarrow y_{s g f}$ as $\omega_{s g} \rightarrow 0$.

Similar formulation can be done for Tiptronic upshifts where the zone will be moving towards right with offset $Y_{p}$.

\section{Identification of Friction Torque Coefficient}

Figure 28 shows phase plane trajectories for different values of friction torque coefficient $b$, generated by backwards in time simulation shown in Figure 16. 
FIGURE 28 Backwards in time simulation for $b=0.08 ; 0.1 ; 0.12 \mathrm{Nm} / \mathrm{rad} / \mathrm{sec}$

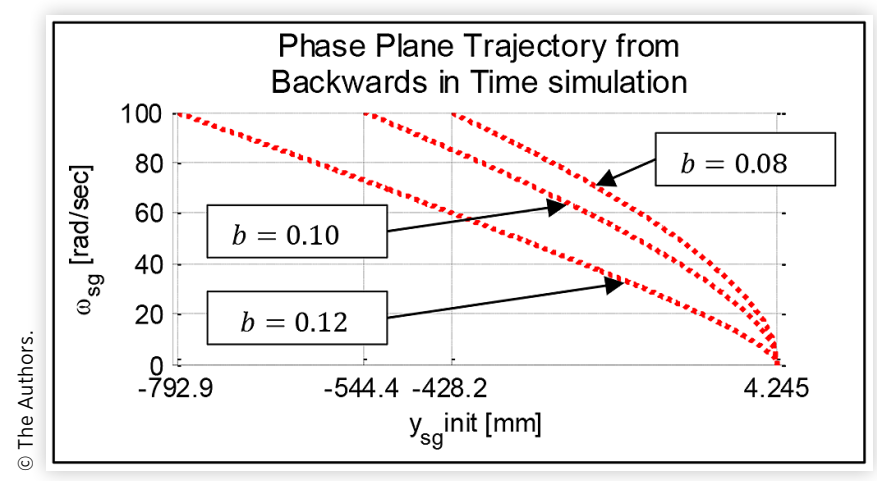

From $\mathrm{x}$-axis in Figure 28 it can be seen that phase plane trajectory is changing very much while friction torque coefficient $b$ is changing $\pm 20 \%$. Since shape of zone used for feedback control depends on $y_{\text {sg_init }}$ trajectory as shown by $\underline{\text { equations } 25}$ and $\underline{28}$, accuracy in value of $b$ used in Figure 16 is very important.

Friction torque coefficient $b$ can be calculated by letting $T_{s}=0$ in equation 7 as shown in [11]. So

$$
b=-J_{g} \dot{\omega}_{g} \div \omega_{g}
$$

Another method can be by putting the idler gear in "Zero acceleration" state shown in Figure 13. In that case friction torque coefficient $b$ can be calculated from equation 7 as

$$
b=T_{s} \div \omega_{g}
$$

But $T_{s}=0$ maneuver is not part of a gear shift and friction torque coefficient changes with changing conditions in transmission as shown in [] ]. Also, duration of Zero acceleration state is typically too small as shown in section "Increase in synchronization time due to feedback control" for accurate readings. Equations 40 and $\underline{41}$ can be used to get an initial estimate of friction torque coefficient.

More accurate value of $b$ for a shift under certain conditions can be calculated after the shift by solution of equation 7. This value of friction torque coefficient can then be used for feedback control when the same shift is scheduled in future under same conditions.

During a down shift, two values of gear velocity $\omega_{i}$ and $\omega_{f}$, time $t_{f}$ apart, are recorded as shown in Figure 29. As shown

\section{FIGURE 29 Data recording for estimation of $b$ from} a downshift

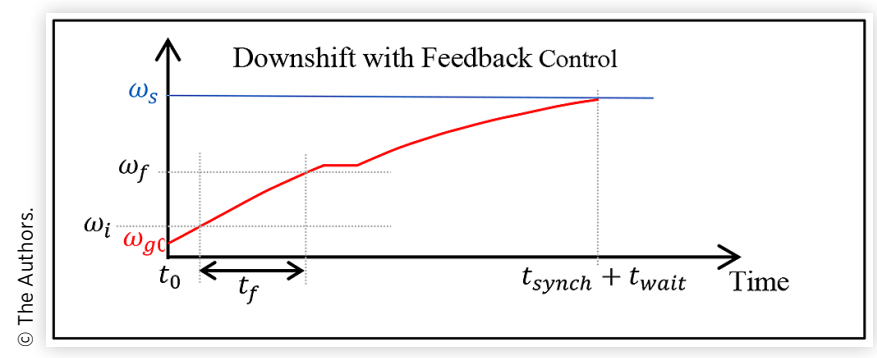

in Figure 29 the two measurements are recorded during Maximum Acceleration state of controller shown in Figure 13.

Using $\omega_{i}, J_{g}, t_{f}$ and $T_{\text {max }}$ in equation 8 along with friction torque coefficient, the resulting final gear velocity $\omega_{f}$ will be

$$
\omega_{f}=\left(T_{\max } / b\right)+\left(\omega_{i}-T_{\max } / b\right) \times e^{-b \times t_{f} / J_{g}}
$$

Equation 42 cannot be solved for $b$ i.e. equation of form 40 or 41 cannot be formulated from equation 42 . To find $b$ a search algorithm needs to be applied, which can give different values of friction torque coefficient to equation 42 and evaluate if resulting idler gear velocity after time $t_{f}$ is equal to $\omega_{f}$.

\section{Limits of Friction Torque Coefficient}

Before the application of search algorithm, it is necessary that the limits of friction torque coefficient $b$ are defined. Since it's a physical quantity, so

$$
b \in\left(0, b_{\max }\right)
$$

Since friction torque can never be actually 0 , hence the use of open start interval in equation 43.

Corresponding to the two limits of $b, \omega_{f}$ in equation 42 will also be limited, so

$$
\omega_{f} \in\left(\omega_{b 0}, \omega_{b \max }\right)
$$
will be

If $b$ is at lower limit i.e. 0 then gear velocity after time $t_{f}$

$$
\omega_{b 0}=\omega_{i}+t_{f} \times T_{\max } \div J_{g}
$$

Gear velocity $\omega_{f}$ will be smaller than $\omega_{b 0}$, since $\omega_{b 0}$ is velocity of a system after a certain time $t_{f}$ that has zero friction torque and $\omega_{f}$ is the velocity of a system after same time $t_{f}$ with finite friction torque coefficient. So

$$
\omega_{f}<\omega_{b 0}
$$

Maximum value of $b$ i.e. $b_{\text {max }}$ is such that the friction is so high that idler gear velocity becomes saturated at $\omega_{f}$. So, if

$$
b_{\max }=T_{\max } \div \omega_{f}
$$

then acceleration of gear $\dot{\omega}_{g}$ becomes 0 in equation 7 describing dynamics of idler gear and gear velocity will not increase further than $\omega_{f}$. As mentioned earlier since $\omega_{f}, \omega_{i}$ and $t_{f}$ are recorded from an actual shift in the past, friction torque coefficient $b$, being equal to $b_{\text {max }}$ is not possible, hence the use of open end interval in equation 43.

If $b$ is at its higher limit then gear velocity after time $t_{f}, \omega_{\text {bmax }}$ can be calculated by putting $b$ equal to $b_{\text {max }}$ in equation 42

$$
\omega_{\text {bmax }}=\omega_{f}+\left(\omega_{i}-\omega_{f}\right) \times e^{-\left(T_{\max } \times t_{f}\right) \div\left(\omega_{f} \times J_{g}\right)}
$$

In equation 48 since $\left(T_{\max } \times t_{f}\right) \div\left(\omega_{f} \times J_{g}\right)$ is $>0$, it follows that $0<e^{-\left(T_{\max } \times t_{f}\right) \div\left(\omega_{f} J_{g}\right)}<1$ and since $\omega_{i}<\omega_{f}$, it follows that

$$
\omega_{f}>\omega_{b \max }
$$

Based on equations 46 and $\underline{49}$, equation 44 must be updated to correspond to the traditional definition of an interval 
FIGURE 30 Idler gear velocity and friction coefficient limits

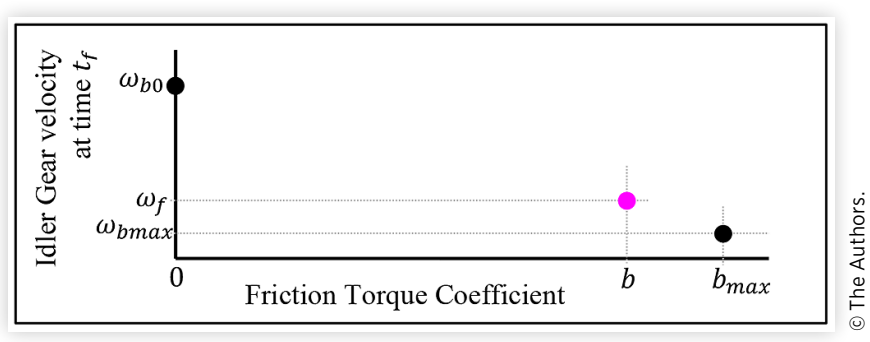

$$
\omega_{f} \in\left(\omega_{b \max }, \omega_{b 0}\right)
$$

$\underline{\text { Figure } 30}$ shows combination of equations 43 and $\underline{50}$.

It can be seen that Figure 30 represents a sorted array since, as friction torque coefficient increases, idler gear velocity at time $t_{f}$ decreases. Binary search algorithm according to [12] can be used on sorted arrays. The aim of using this particular search algorithm is to demonstrate the usage of search algorithms on this particular problem. Usage of other search algorithms and their performance is a topic for future research.

\section{Binary Search Algorithm}

The principle of binary search algorithm is to first define an interval $\left[b_{\text {low }}, b_{\text {high }}\right]$ such that $b \in\left[b_{\text {low }}, b_{\text {high }}\right]$. Then it calculates a friction torque coefficient $b_{\text {mid }}$, which is the middle value of the search interval $\left[b_{\text {low }}, b_{\text {high }}\right]$. According to equation 43 the search interval is $\left(0, b_{\text {max }}\right)$ shown by dotted blue square in Figure 31 . So $b_{\text {mid }}$ will be $b_{\text {max }} / 2$, as shown by the red dot.

Then $\omega_{\text {fmid }}$ is calculated for $b_{\text {mid }}$ using 42 . Then by comparing $\omega_{\text {fmid }}$ with $\omega_{f}$, it can be judged whether $b \in\left[b_{\text {mid }}, b_{\text {high }}\right]$ OR $b \in\left[b_{\text {low }}, b_{\text {mid }}\right]$. In the particular case shown in Figure 31 since $\omega_{\text {fmid }}>\omega_{f}$ and also since the Figure 31 is a sorted array, it can be concluded that $b \in\left[b_{\text {mid }}, b_{\text {high }}\right]$.

In next iteration, $b_{\text {low }}$ is changed to the lower value of search interval from previous iteration i.e. $b_{\text {mid }}$ and a new value of $b_{\text {mid }}$ is calculated as shown in Figure 32.

Then a new value of $\omega_{\text {fmid }}$ is calculated again as shown in Figure 32.It can be seen from Figure 32, that $b_{\text {mid }}$ has come quite close to the unknown $b$, in just two iterations and it can be concluded that with more iterations $b_{\text {mid }}$ will come closer and closer to $b$. The complete binary search algorithm is shown in Figure 33

\section{FIGURE $31 \quad 1^{\text {st }}$ iteration of Binary search algorithm}

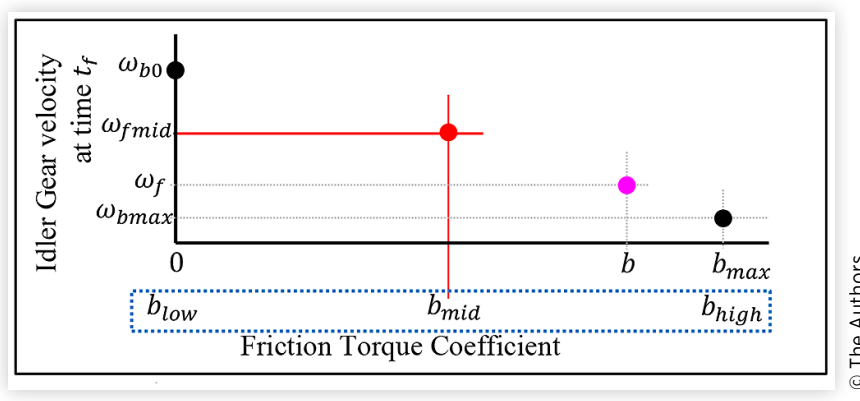

\section{FIGURE $322^{\text {nd }}$ iteration of Binary Search algorithm}

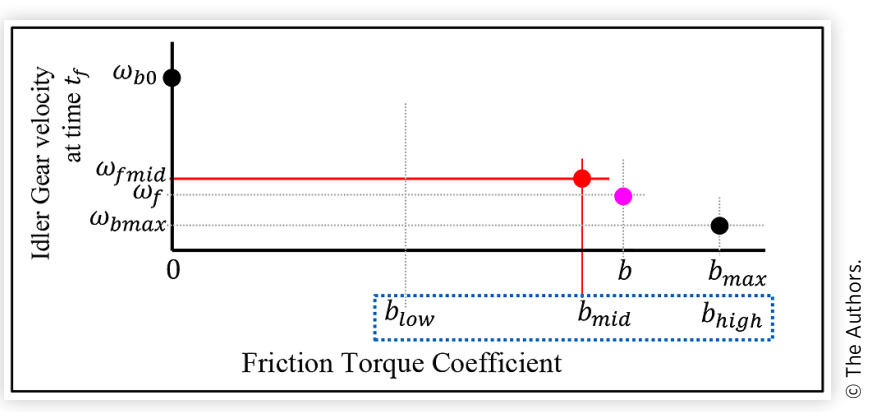

FIGURE 33 Binary Search algorithm

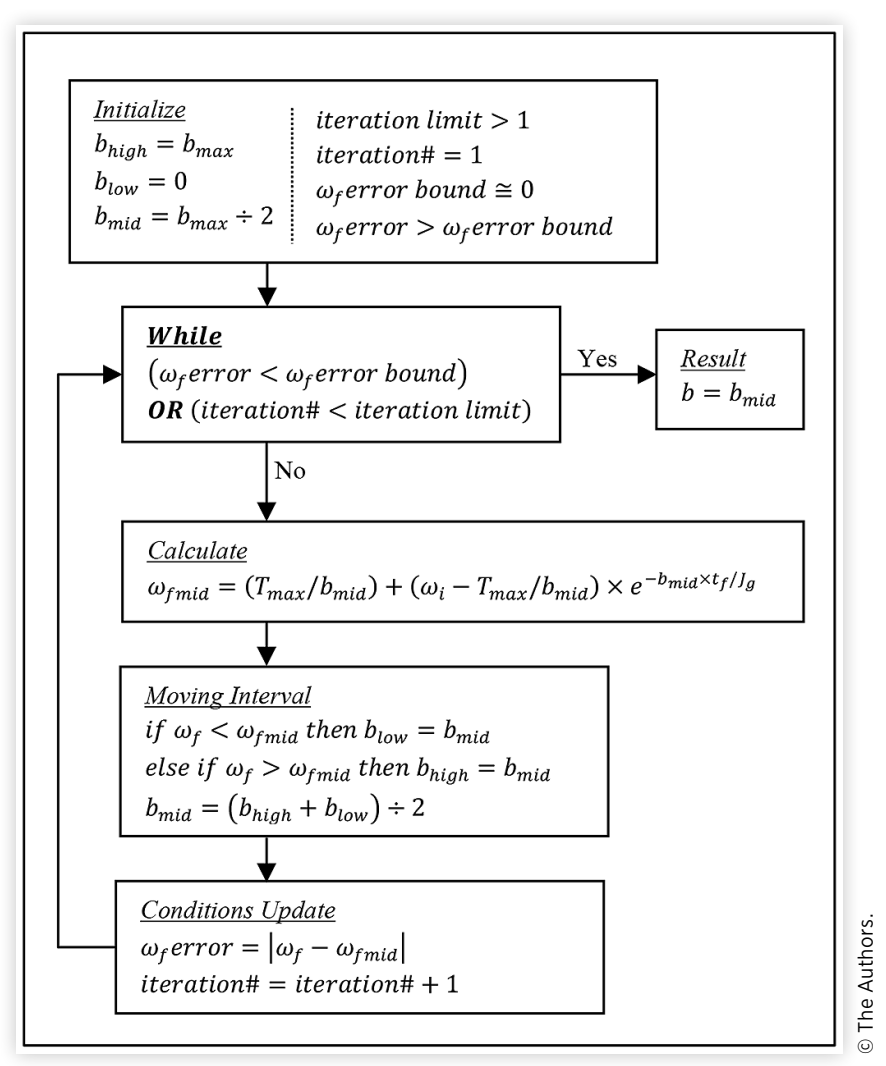

Since the algorithm is in a "While" loop, the break conditions need to be specified carefully. As shown in Figure 33, the while loop is broken if $\omega_{\text {fmid }}$ is sufficiently close to $\omega_{f}$. This condition is defined by $\omega_{f}$ error bound parameter in "Initialize" block. If $\omega_{f}$ error defined in "Conditions Update" block is smaller than $\omega_{f}$ error bound, the while loop is broken. The while loop is also broken if number of iterations shown by iteration\# in "Conditions Update" block larger than an arbitrarily large iteration limit defined in "Initialize" block.

\section{Test of Binary Search Algorithm}

Input parameters to binary search algorithm are shown in Table 4. 
TABLE 4 Input to Binary Search algorithm

\begin{tabular}{|l|l|}
\hline \multicolumn{2}{|l|}{ Input Parameters to Binary Search Algorithm } \\
\hline$\omega_{i}$ & $110 \mathrm{rad} / \mathrm{sec}$ \\
\hline$\omega_{f}$ & $150 \mathrm{rad} / \mathrm{sec}$ \\
\hline$t_{f}$ & $67.3 \mathrm{~ms}$ \\
\hline$b_{\max }$ & $0.1667 \mathrm{Nm} / \mathrm{rad} / \mathrm{sec}$ \\
\hline$\omega_{b 0}$ & $194.1181 \mathrm{rad} / \mathrm{sec}$ \\
\hline$\omega_{b \max }$ & $127.1696 \mathrm{rad} / \mathrm{sec}$ \\
\hline iteration limit & 20 \\
\hline$\omega_{f}$ error bound & $0.01 \mathrm{rad} / \mathrm{sec}$ \\
\hline
\end{tabular}

Value of friction torque coefficient $b$ is $0.1 \mathrm{Nm} / \mathrm{rad} / \mathrm{sec}$ according to Table 1 . Figure 34 shows convergence of binary search algorithm. On $\mathrm{x}$ axis the iterations are shown. On $\mathrm{y}$-axis, for each iteration the value of $b_{\text {high }}$ and $b_{\text {low }}$ are shown with blue dots and value of $b_{\text {mid }}$ is shown by red crosses. From Figure 34, it can seen that after 12 iterations, the algorithm stops and comes quite close to value of $b$ equal to $0.1 \mathrm{Nm} / \mathrm{rad} / \mathrm{sec}$.

After 12 iterations, $\omega_{f}$ error is $0.0061 \mathrm{rad} / \mathrm{sec}$ hence becoming less than $\omega_{f}$ error bound and the search algorithm stops.

Figure 35 shows the zoomed in view and last 3 iterations. It can be seen how close $b_{\text {mid }}$ is to $b$. The difference between $b_{\text {mid }}$ of $12^{\text {th }}$ iteration and $b$ is $-1.6276 \mathrm{e}-05 \mathrm{Nm} / \mathrm{rad} / \mathrm{sec}$, which is negligible.

\section{FIGURE 34 Convergence of Binary Search algorithm}

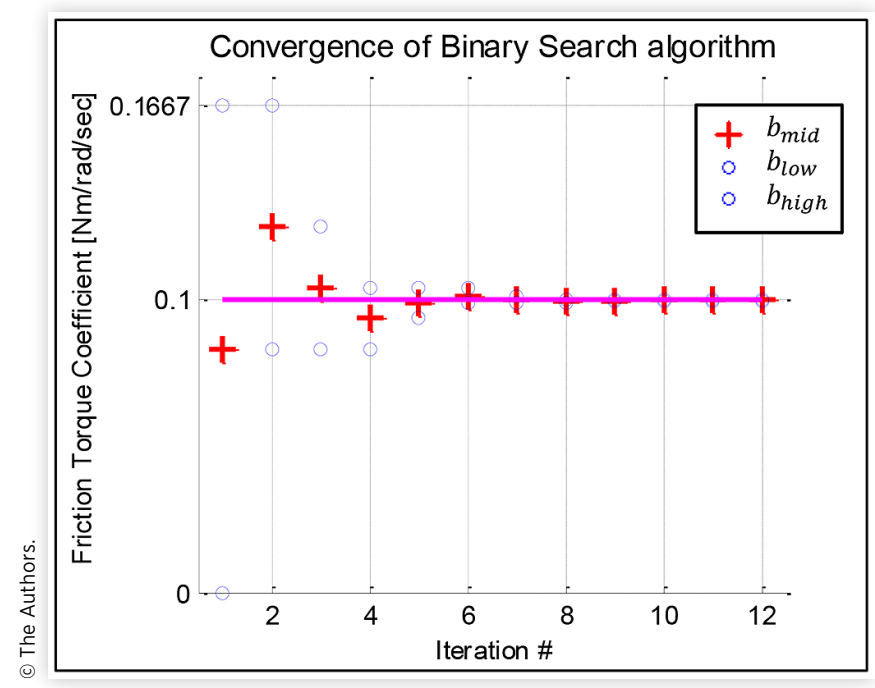

FIGURE 35 Final Iterations of Binary search algorithm

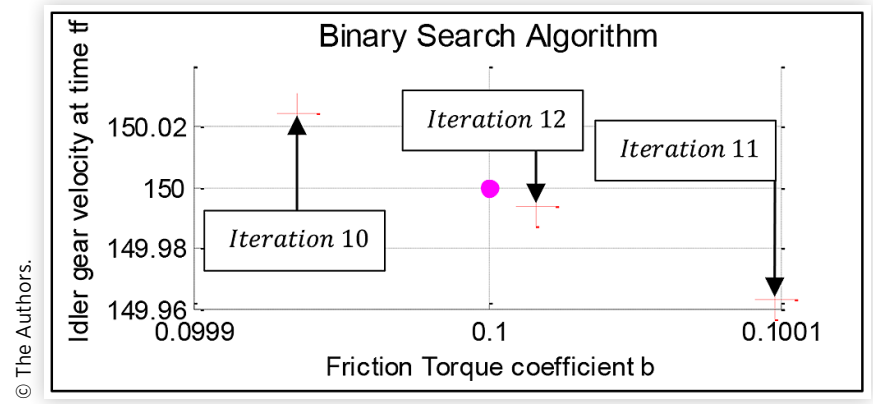

The resulting friction torque coefficient $b$, will then be recorded along with conditions in transmission such as oil temperature etc. By repeating this process after many shifts, the transmission will have a database of accurate friction torque coefficients for different conditions. In the future when a shift is scheduled the friction torque coefficient then used in Backwards in time simulation shown in Figure 16 will be an accurate estimate.

In future, this approach also has potential for predictive maintenance. If for example after a shift under known conditions the value of $b$ is very different from previously identified value, it might be an indication that there is some issue in the transmission.

\section{Conclusions}

This paper has presented detailed design recipe for a modelbased feedback controller for synchronizer systems to guarantee minimum noise and wear and fast gear engagement during gear shifts. All the parameters in the feedback control system are based on physical parameters of transmission system, so adapting this control algorithm for any transmission is quite easy. The aim of control design in this paper is not to achieve theoretical optimum but rather to be close to optimal while fitting within software and hardware limits of real DCTH.

The parts of feedback control that are to run during gear shifts are kept very simple. A few conditional statements and 2 look up tables are all that is required while feedback control is running during shifts. All the complex mathematical operations can be done offline or when the transmission controller is free. This saves a lot of computation power and memory.

It is shown by simulation that the feedback controller can improve gear shift quality while adding negligible time to gear shift process.

Adaptation to the algorithm required to control Tiptronic transmissions is also discussed.

Method for identification of friction torque coefficient is described in detail. Binary search algorithm is used to identify the friction torque coefficient and it is shown that the algorithm can identify friction torque coefficient quite quickly and with a very high accuracy. With the method presented, feedback controller can adapt to varying friction in transmission rather than relying on offline calibration values. The identification method has potential to be used for predictive maintenance and it will be a topic for future research.

This paper shows that it is theoretically possible to achieve a fast and noise free gearshift control, however the effect of sensor and actuator delays and errors are not analyzed in detail. The effects of these delays on the control algorithm will be a part of future research.

\section{References}

1. Piracha, M.Z., Grauers, A., and Hellsing, J., "Improving Gear Shift Quality in a PHEV DCT with Integrated PMSM," in CTI Symposium Automotive Transmissions, HEV and EV Drives, Berlin, 2017. 
2. Tseng, C.-Y. and Yu, C.-H., "Advanced Shifting Control of Synchronizer Mechanisms for Clutchless Automatic Manual Transmission in an Electric Vehicle," Mechanism and Machine Theory 84:37-56, 2015.

3. Penta, A., Gaidhani, R., Sathiaseelan, S.K., and Warule, P., "Improvement in Shift Quality in a Multi Speed Gearbox of an Electric Vehicle through Synchronizer Location Optimization," SAE Technical Paper 2017-01-1596, 2017, doi:https://doi.org/10.4271/2017-01-1596.

4. Lu, Z., Chen, H., Wang, L., and Tian, G., “The Engaging Process Model of Sleeve and Teeth Ring with a Precise, Continuous and Nonlinear Damping Impact Model in Mechanical Transmissions," SAE Technical Paper 2017-012443, 2017, doi:https://doi.org/10.4271/2017-01-2443.

5. Chen, H. and Tian, G., "Modeling and Analysis of Engaging Process of Automated Mechanical Transmissions," Multibody System Dynamics 37:345-369, 2016.

6. Hoshino, H., "Analysis on Synchronization Mechanism of Transmission," in 1999 Transmission and Driveline Systems Symposium, 1999.

7. Piracha, M.Z., Grauers, A., Barrientos, E., Budacs, H. et al., "Model Based Control of Synchronizers for Reducing Impacts during Sleeve to Gear Engagement," SAE Technical Paper 2019-01-1303, 2019, doi:https://doi.org/10.4271/2019$\underline{01-1303 .}$.
8. Math, K.M.H. and Lund, M., "Drag Torque and Synchronization Modelling in a Dual Clutch Transmission," Chalmers University of Technology, Gothenburg, Sweden, 2018.

9. Walker, P.D. and Zhang, N., "Engagement and Control of Synchronizer Mechanisms in Dual Clutch Transmissions," Journal of Mechanical Systems and Signal Processing 26:320332, 2012.

10. Naidu, D.S., Optimal Cotrol Problems (Pocatello, Idaho: CRC Press, 2003), 293-314.

11. Blessing, U.C., Schuerlein, M., and Meissner, J., "Method for Adapting the Kiss Point," U.S. Patent US8577573B2, Nov. 05, 2013.

12. Cormen, T.H., Leiserson, C.E., Rivest, R.L., and Stein, C., Introduction to Algorithms (MIT Press, 2009).

13. Duan, C., "Analytical Study of a Dog Clutch in Automatic Transmission Application," Internation Journal of Passengar Cars, Mechanical Systems 7(3):1155-1162, 2014.

\section{Contact Information}

\section{Muddassar Zahid Piracha}

muddassar.piracha@cevt.se +46721843970

(c) 2020 The Authors. Published by SAE International. This Open Access article is published under the terms of the Creative Commons Attribution License (http:// creativecommons.org/licenses/by/4.0/), which permits distribution, and reproduction in any medium, provided that the original author(s) and the source are credited. 